\title{
Preliminary scattering kernels for ethane and triphenylmethane at cryogenic temperatures
}

\author{
F. Cantargi ${ }^{a}$, J.R. Granada, and J.I. Márquez Damián \\ Centro Atómico Bariloche and Instituto Balseiro, Comisión Nacional de Energía Atómica, Argentina
}

\begin{abstract}
Two potential cold moderator materials were studied: ethane and triphenylmethane. The first one, ethane $\left(\mathrm{C}_{2} \mathrm{H}_{6}\right)$, is an organic compound which is very interesting from the neutronic point of view, in some respects better than liquid methane to produce subthermal neutrons, not only because it remains in liquid phase through a wider temperature range $\left(\mathrm{T}_{f}=90.4 \mathrm{~K}, \mathrm{~T}_{b}=184.6 \mathrm{~K}\right)$, but also because of its high protonic density together with its frequency spectrum with a low rotational energy band. Another material, Triphenylmethane is an hydrocarbon with formula $\mathrm{C}_{1} 9 \mathrm{H}_{16}$ which has already been proposed as a good candidate for a cold moderator. Following one of the main research topics of the Neutron Physics Department of Centro Atómico Bariloche, we present here two ways to estimate the frequency spectrum which is needed to feed the NJOY nuclear data processing system in order to generate the scattering law of each desired material. For ethane, computer simulations of molecular dynamics were done, while for triphenylmethane existing experimental and calculated data were used to produce a new scattering kernel. With these models, cross section libraries were generated, and applied to neutron spectra calculation.
\end{abstract}

\section{Introduction}

In both nuclear reactors and accelerator based neutron sources, those particles are produced with energies in the range of MeV's through some form of nuclear reactions. To investigate condensed matter properties through scattering experiments, those neutrons must be slowed down to energies comparable to the excitations and wavelengths similar to the characteristic lengths in the sample.

Most neutron sources are equipped with moderators $(\mathrm{H} 2 \mathrm{O}, \mathrm{D} 2 \mathrm{O},[\mathrm{CH} 2] \mathrm{n})$ at room temperature to produce thermal neutrons, and cryogenic liquids like $\mathrm{H}_{2}$ and $\mathrm{D}_{2}$ to generate cold neutrons. There are several reasons for such preferences, mostly related to maintain a balance amongst the best coupling to the fast neutron source, optimal spectral condition of thermalized neutrons, and the integrity of the moderator's molecular material under very strong radiation fields. The latter is usually a severe constrain for cold neutron moderators operating around $20 \mathrm{~K}$, where most materials become solid and heat removal together with bond breaking processes pose serious practical problems.

In the case of accelerator based pulsed neutron sources, a full exploitation of its time structure in many situations demands a sharp neutron pulse to achieve good resolution in time-of-flight experiments. Consequently, hydrogenous moderators are usually employed at the temperature appropriate to emit neutrons with the required energy spectrum. On purely neutronic properties, like protonic density $(\mathrm{NH})$ and pulse width ( $\left.\mathrm{v} \Delta \mathrm{t}^{1 / 2}\right)$, polyethylene is the best room temperature moderator, and methane the best cold neutron moderator. None of them can be used at a powerful accelerator source for the reasons mentioned above, and therefore water and liquid hydrogen are the traditional choices for those regimes.

Although in other rather special applications very cold and ultra cold neutrons are needed, in which cases deuterated materials are employed on account of the absorption cross section that prevent the use of protonated moderators at those energy regions, there are many other more common applications where neutrons with an effective temperature halfway between those (cold, thermal) referred above are needed.

One of the main research lines of the Neutron Physics Department of Centro Atómico Bariloche is the study of thermal and cold moderator materials. Amongst the latter, cross-section libraries for hydrogen bounded in liquid hydrogen, liquid and solid deuterium, solid methane (phase II), mesitylene, toluene and a mix of them at several temperatures, were already generated, validated and used in calculations to analyze their neutron production [1].

Following this line we present here the study of two other materials: solid triphenylmethane and liquid ethane. At different temperatures and for different reasons, both look as potential convenient moderator material to produce cold neutrons. In this work we present the development of scattering kernels, the generation of their scattering laws and the resulting cross section libraries.

Finally, we indicate the results of preliminary spectra calculations to compare the production of those materials with other standard moderators.

The conceptual ideas and first results for ethane and triphenylmethane were presented elsewhere [2].

a e-mail: cantargi@cab.cnea.gov.ar 


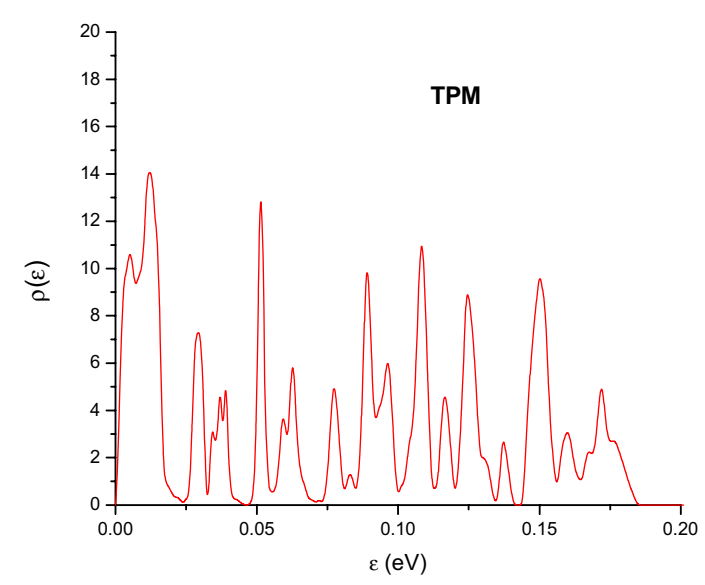

Figure 1. Frequency spectrum based on experimental information used for the generation of triphenylmethane thermal cross section library.

\section{Generation of thermal scattering law libraries}

Within the Gaussian approximation [3], the conventional route to the calculation of the Scattering Law $S(\mathbf{Q}, \omega)$ for a given material involves the characterization of its dynamics through a generalized frequency spectrum $\rho(\varepsilon)$. Such information can be obtained from experimental data, from theoretical models or numerical simulations, or a combination of those. Once $\rho(\varepsilon)$ has been established, it is used to feed the LEAPR module of the NJOY nuclear data processing system [4], which employs a phonon expansion and the incoherent approximation to generate the inelastic cross section. This procedure is able to produce fairly accurate results in the case of hydrogeneous materials, where the incoherent contributions dominate the cross sections [5].

\subsection{Triphenylmethane}

At room temperature, triphenylmethane $\left(\mathrm{C}_{19} \mathrm{H}_{16}\right)$ is a colorless solid, which has already been proposed as a good cold moderator due to it high protonic density and good radiaton resistance. In the same way it was done with the group of hydrocarbons already mentioned, the frequency spectrum for triphenylmethane (TPhM), was constructed using experimental information [6]. In Fig. 1 it is shown the frequency spectrum used for calculations.

In addition to the continuous vibrational spectrum shown in Fig. 1 we included intravibrational modes at frequencies $0.18 \mathrm{eV}$ and $0.37 \mathrm{eV}$ with weights 0.299 and 0.304 , respectively. The mode at $0.18 \mathrm{eV}$ is the $\mathrm{C}-\mathrm{H}$ stretching associated to the central $\mathrm{H} \mathrm{[7]} \mathrm{and} 0.37 \mathrm{eVis}$ the $\mathrm{C}-\mathrm{H}$ stretching in the ring [8].

The NJOY code was employed to evaluate the scattering law and preliminary cross section libraries at different temperatures of interest.

The total scattering cross section of ( $\mathrm{H}$ in) Triphenylmethane is compared in Fig. 2 with other solid moderator materials at $20 \mathrm{~K}$. The macroscopic inelastic scattering cross sections of those materials are shown in Fig. 3, where we can observe that the behavior of TPhM is the one that approaches methane amongst the set of materials considered here.

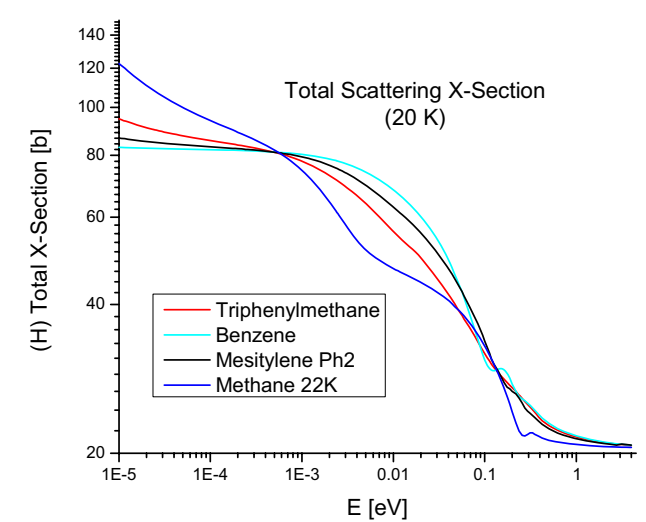

Figure 2. Total cross section for different moderator materials.

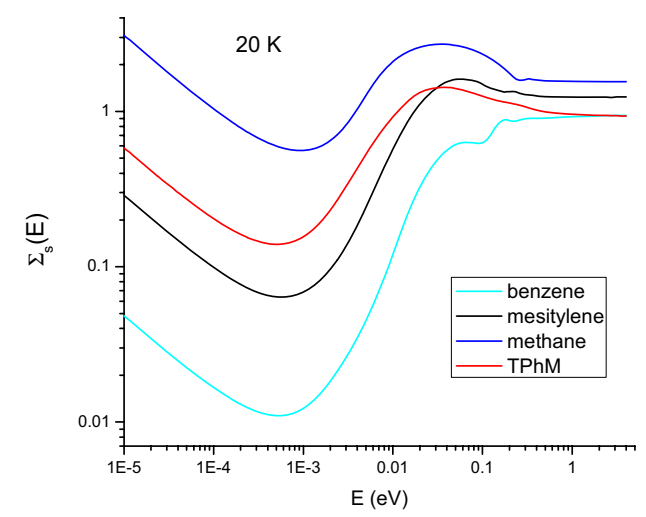

Figure 3. Macroscopic inelastic cross sections for different solid moderator materials at $20 \mathrm{~K}$.

Table 1. Neutronic properties of some common moderator materials.

\begin{tabular}{|c|c|c|c|c|c|c|}
\hline Parameter & $\begin{array}{l}\mathrm{H}_{2} \mathrm{O} \\
293 \mathrm{~K}\end{array}$ & $\begin{array}{l}\left(\mathrm{CH}_{2}\right)_{n} \\
293 \mathrm{~K}\end{array}$ & $\begin{array}{l}\mathrm{CH}_{4} \\
20 \mathrm{~K}\end{array}$ & $\begin{array}{c}\mathrm{CH}_{4} \\
100 \mathrm{~K}\end{array}$ & $\begin{array}{l}\mathrm{C}_{2} \mathrm{H}_{6} \\
100 \mathrm{~K}\end{array}$ & $\begin{array}{l}\mathrm{C}_{2} \mathrm{H}_{6} \\
180 \mathrm{~K}\end{array}$ \\
\hline$\sigma_{\text {firee }}$ (barn) & 44.749 & 45.73 & 86.73 & 86.73 & 132.46 & 132.46 \\
\hline $\begin{array}{ll}\rho & \left(\mathrm{g} / \mathrm{cm}^{3}\right) \\
\end{array}$ & 1 & 0.94 & 0.510 & 0.4392 & 0.641 & 0.550 \\
\hline$N^{\text {molec }}\left(10^{22} / \mathrm{cm}^{3}\right)$ & 0.0335 & 0.0404 & 0.0192 & 0.0165 & 0.0129 & 0.0110 \\
\hline $\begin{array}{ll}N^{H} & \left(10^{22} / \mathrm{cm}^{3}\right)\end{array}$ & 6.69 & 8.09 & 7.68 & 6.61 & 7.72 & 6.63 \\
\hline D $\quad\left(100^{-5} \mathrm{~cm}^{2} / \mathrm{s}\right)$ & 2.3 & - & -- & 5.2 & 0.82 & 5.51 \\
\hline$\Sigma_{\text {free }}\left(\mathrm{cm}^{-1}\right)$ & 1.5 & 1.849 & 1.665 & 1.431 & 1.705 & 1.463 \\
\hline$\xi$ & 0.926 & 0.913 & 0.954 & 0.954 & 0.940 & 0.940 \\
\hline$\xi \sum_{\text {tree }}\left(\mathrm{cm}^{-1}\right)$ & 1.39 & 1.69 & 1.59 & 1.37 & 1.60 & 1.38 \\
\hline $\bar{y}$ & 0.924 & 0.908 & 0.951 & 0.951 & 0.936 & 0.936 \\
\hline $\begin{array}{ll}v t_{s} & (\mathrm{~cm}) \\
\end{array}$ & 2.105 & 1.723 & 1.858 & 2.162 & 1.832 & 2.135 \\
\hline$v \Delta t_{s}(\mathrm{~cm})$ & 1.183 & 0.963 & 1.055 & 1.227 & 1.034 & 1.205 \\
\hline$v \Delta t_{1 / 2}(\mathrm{~cm})$ & 2.346 & 1.915 & 2.080 & 2.421 & 2.046 & 2.383 \\
\hline
\end{tabular}

\subsection{Ethane}

Ethane is a hydrocarbon with chemical formula $\mathrm{C}_{2} \mathrm{H}_{6}$, that can be viewed as a joint of two methyl groups. Of particular interest from the point of view of moderator material is its existence as a liquid over a large temperature range $\left(\mathrm{T}_{\mathrm{f}}=90.4 \mathrm{~K}, \mathrm{~T}_{\mathrm{b}}=184.6 \mathrm{~K}\right)$, while its protonic density is still very high. Other neutronic properties of liquid ethane are given in Table 1, where data from standard moderators are also included [11].

Most quantities in Table 1 have a clear meaning, while $\xi$ is the logarithmic energy decrement, and $\mathrm{t}_{s}, \Delta \mathrm{t}_{s}$ and $\Delta \mathrm{t}_{1 / 2}$ represent the slowing down time, its standard deviation, and the FWHM of the thermalized neutron pulse, respectively. It is clear from Table 1 that besides the solid materials, liquid ethane has similar or better properties than light water.

The actual protonic density of ethane over its liquid range is shown in Fig. 4, compared to those of other 


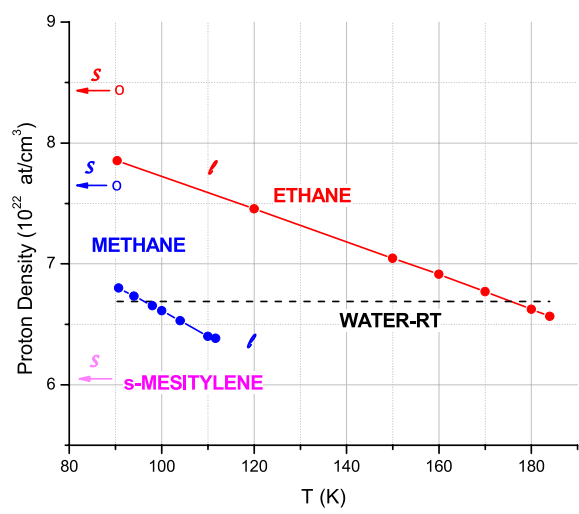

Figure 4. The protonic density of some traditional moderators over the liquid range of ethane.

common moderators. It is observed that such range is as large $(\sim 100 \mathrm{~K})$ as that for liquid water, but $200 \mathrm{~K}$ lower. On the other hand, the liquid ethane density varies there roughly in between the values for solid and liquid methane, the latter existing over a much shorter range $\left(T_{f}=90.7 \mathrm{~K}\right.$, $\left.\mathrm{T}_{\mathrm{b}}=111.7 \mathrm{~K}\right)$.

In this case, for building the frequency spectrum of liquid ethane, molecular dynamics simulations based on the code GROMACS [10] were done to calculate the time evolution of the molecules. An ensemble of 512 molecules interacting via the OPLS-AA potential [11] was employed. The system was equilibrated at $\mathrm{T}=$ $100 \mathrm{~K}, \mathrm{P}=1$ bar over $6 \mathrm{nsec}$ with 0.1 fsec steps, using the Nosé-Hoover algorithm for the barostat and the ParrinelloRahman for the thermostat.

The total simulation time was $40 \mathrm{ps}$, which is long enough to allow the long time diffusional dynamics to appear, with a time step of $0.1 \mathrm{fs}$. A vector including the velocities of each atom was saved every 10 frames into a trajectory file, afterwards processed to compute the velocity autocorrelation function (VACF)

$$
\operatorname{VACF}(\tau)=<v(t) E v(t+\tau)>.
$$

Finally, the frequency spectrum was computed from the molecular dynamics results by taking the Fourier transform of the VACF Fig. 5.

The frequency spectrum thus obtained is shown in

$$
\rho(\epsilon)=\frac{2 M}{3 k T h}\left|\sum_{k=0}^{N-1} V A C F\left(\tau_{n}\right) e^{2 \pi k_{n} / N}\right| \Delta \tau
$$

For the NJOY calculation the generalized frequency spectrum was split in two parts:

a) A diffusive component described by the EgelstaffSchofield model with an effective mass $\mathbf{M}_{\text {diff }}=$ $3 \mathrm{M}_{m o l}$, and a self-diffusion coefficient $\mathrm{D}=0.71 \times$ $10^{-5} \mathrm{~cm}^{2} / \mathrm{s}$ [12],

b) A continuous part containing the rotational and internal vibrational modes of the ethane molecule.

With such continuous spectrum, the inclusion of two discrete oscillators at $0.18 \mathrm{eV}$ and $0.37 \mathrm{eV}$ corresponding to the $\mathrm{H}$ vibrations within the methyl groups, and the diffusive term with the self-coefficient value at the calculation temperature, we obtain the scattering laws and

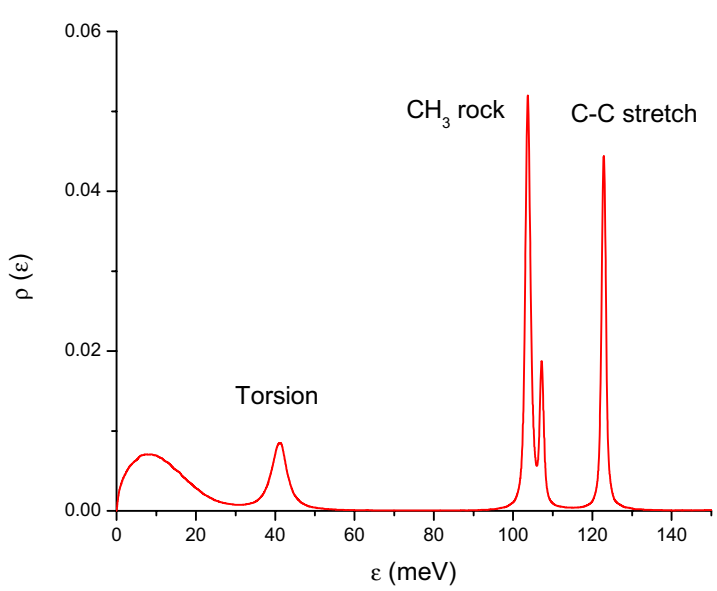

Figure 5. The continuous part of the frequency spectrum obtained from a GROMACS MD calculation for liquid ethane.

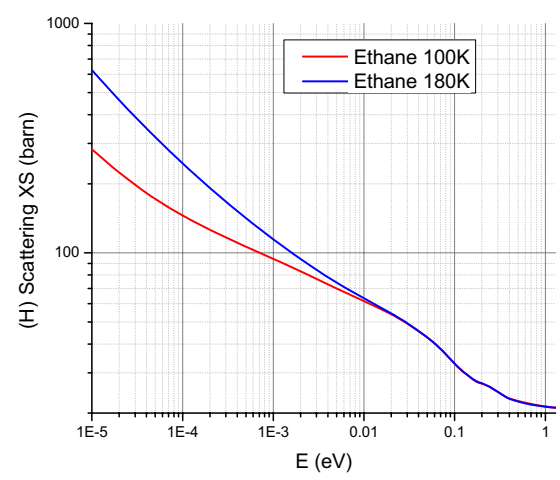

Figure 6. The total scattering of $(\mathrm{H}$ in) liquid ethane at $100 \mathrm{~K}$ and $180 \mathrm{~K}$.

the cross section libraries for liquid ethane at different temperatures. The total scattering cross sections at $100 \mathrm{~K}$ and $180 \mathrm{~K}$ are shown in Fig. 6.

It is evident in Fig. 6 the difference between the two curves for neutron energies below $\sim 0.02 \mathrm{eV}$, due to the thermally induced excitation of the low-energy rotational modes and the increase of the self-diffusion coefficient by a factor 8.5 as the temperature increases from $100 \mathrm{~K}$ to $180 \mathrm{~K}$.

\section{Results}

In order to make a preliminary assessment of the neutron production performance, we performed spectra calculations using our new libraries in a Monte Carlo code [13], for decoupled slab-type moderators of $12 \times 12 \times$ $5 \mathrm{~cm}^{3}$.

We show in Fig. 7 the neutron emission spectra for triphenylmethane together with other common moderators at $20 \mathrm{~K}$. As can be seen, the intensity produced by $\mathrm{TPhM}$ seems to be only second to methane, but it must be emphasized that these are just preliminary evaluations, that need to be contrasted with experimental results.

The results for liquid ethane are shown in Fig. 8. Although not necessarily the production from different materials is optimized for that particular geometry, it is very interesting to note that liquid ethane at $100 \mathrm{~K}$ seems to produce a neutron flux larger than liquid methane at $100 \mathrm{~K}$, at all energies from thermal downwards. 


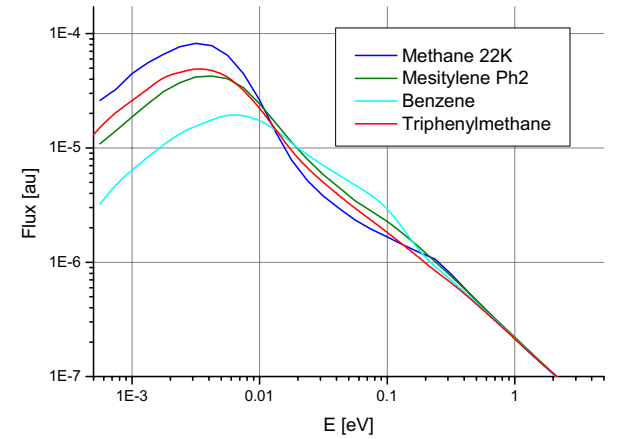

Figure 7. Emission spectra for different moderator materials at $\sim 20 \mathrm{~K}$.

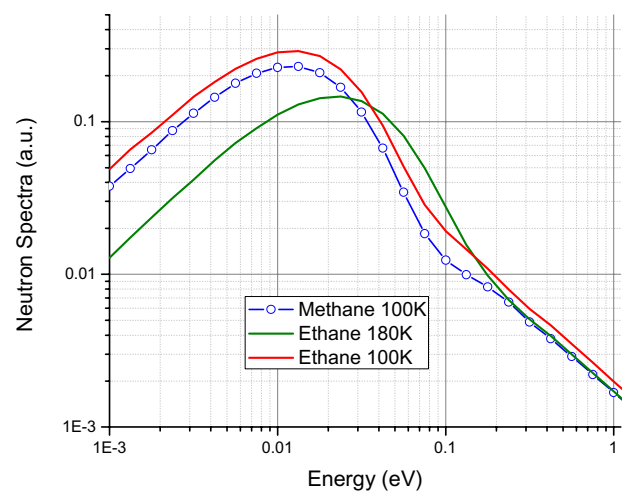

Figure 8. Emission spectra for liquid ethane at $100 \mathrm{~K}$ and $180 \mathrm{~K}$, together with methane at $100 \mathrm{~K}$.

\section{Conclusions}

This work involved a preliminary exploration of the neutronic properties of solid triphenylmethane and liquid ethane as potential moderators to produce cold and subthermal neutrons, respectively. In order to confirm that possibility it is imperative to perform transmission and spectra measurements, as there is a lack of that kind of data for both materials. This is also true for ethane except at low temperature [14], where there is no better hydrogenous moderator material than solid methane or liquid hydrogen in the case of strong radiation fields.
Liquid methane at $100 \mathrm{~K}$ has been used as a moderator to produce subthermal neutrons in several facilities, but it is possible that liquid ethane at that temperature could give an increased production at neutron energies in between thermal and cold regions.

\section{References}

[1] http://www.cab.cnea.gov.ar/nyr/tsl_eng . html

[2] J.R. Granada et al., IAEA CRP 1916 / F1.20.26 "Advanced Moderators for Intense Cold Neutron Beams in Materials Research", Vienna, 22-26 Feb. (2016)

[3] G.L. Squires, Thermal Neutron Scattering, Cambridge University Press (1978)

[4] R.E. MacFarlane, D.W. Muir, The NJOY Nuclear Data processing System, Los Alamos National Laboratory Report LA-12740-M (1994)

[5] J.I. Márquez Damián et al., Nuclear Data Sheets 118, 169 (2014)

[6] I. Majerz and I. Natkaniec, Physica B 350, e439e442 (2004)

[7] Th. Hügle, M. Mocko, M.A. Hartl, L.L. Daemen, G. Muhrer, NIMA 738, 1-5 (2014)

[8] F. Cantargi, J.R. Granada, NIMB 268, 2487-2491 (2010)

[9] J.R. Granada et al., Thermal neutron cross section libraries for liquid Ethane, UCANS-VI, Xi' an, China (25-28 Oct 2016).

[10] GROMACS (http://www.gromacs.org/)

[11] Jorgensen, William L., David S. Maxwell, and Julian Tirado-Rives. Development and testing of the OPLS all-atom force field on conformational energetics and properties of organic liquids. Journal of the American Chemical Society 118(45), 11225-11236 (1996)

[12] J.F. Harmon, B.H. Muller, Phys. Rev. 182, 400 (1969)

[13] MCNP - A General Monte Carlo N-Particle Transport Code - Version 5. X-5 Monte Carlo Team, Los Alamos National Laboratory (2003)

[14] K. Inoue et al., J.At. En. Soc. Japan 21, 865 (1979) 